\title{
Jagged edge
}

It's highly debatable whether these sharp-toothed ribbons will ever be put to use as blades for nanoscale saws. But the wonder is that they form at all. Made from semiconducting cadmium selenide (CdSe), they have one edge that is atomically smooth whereas the other traces out a sawtooth shape reminiscent of a graph of random noise.

These structures, just a few micrometres wide, are produced by evaporating CdSe powder in a furnace and using a flow of nitrogen to carry the vapour to a cooler region strewn with gold nanoparticles
(C. Ma et al. J. Am. Chem. Soc. doi:10.1021/ja0395644; 2003). The gold particles act as catalysts for the regrowth of CdSe crystals as ribbonshaped structures.

Ma et al. think that the asymmetry of the ribbon edges one smooth, the other jagged - is caused by their differing chemical composition. The orientation of crystal planes in the ribbons means that their edges terminate in a layer of either cadmium or selenium. The former is more chemically reactive: in zinc oxide (which has the same crystal structure), a zinc-capped surface can act as a self-catalyst for

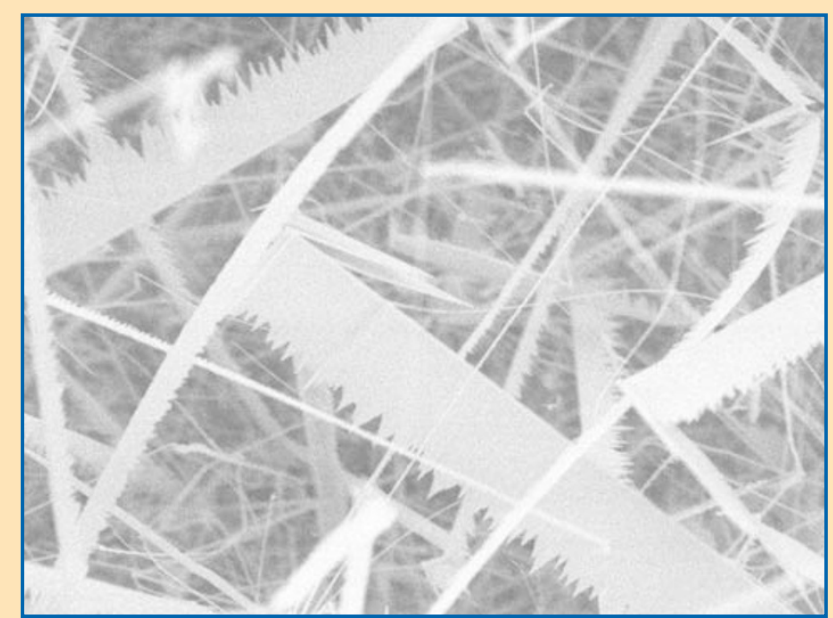

further growth, resulting in comblike structures (Z. L. Wang et al. Phys. Rev. Lett. 91, 185502; 2003). A similar process here at cadmium- terminated edges produces lateral growth with a rough profile, which resolves itself into the sawtooth profile. Philip Ball to separate efficiently. So the authors screened for genes that were lethal only when expressed with Rec8 in mitotic fission yeast. They isolated one such gene, which is usually expressed only in meiosis, and named its encoded protein Sgo1, short for 'Sugoshin' — 'guardian spirit' in Japanese.

Katajima et al. found that expressing Sgo 1 with Rec8 in mitotic cells allowed Rec8 to persist at mitotic centromeres. Moreover, Sgol associates with centromeres in early meiosis and remains there until anaphase I, as expected (Fig. 2). Deleting the gene results in normal chromosome segregation in meiosis I but random segregation of sister chromatids in meiosis II, because of premature loss of cohesion at anaphase I (because all Rec8 is now cleaved). The authors further show that Sgol and Rec8 associate in the same complex. These data are consistent with a role for Sgol in protecting centromeric Rec8 from separase at the onset of anaphase I.

How is Sgol recruited to centromeres? Here, too, Kitajima et al. suggest an answer. During both meiosis and mitosis, a 'checkpoint' monitors the attachment of centromeres to the spindle - the apparatus that physically separates chromosomes. This checkpoint also monitors the physical tension between sister centromeres. By doing so it ensures that sister chromatids make the correct attachments and achieve the required arrangement on the spindle ${ }^{9}$. Previous analyses of fission yeast ${ }^{10}$ had shown that one checkpoint component, $\mathrm{Bub} 1$, is required to maintain $\operatorname{Rec} 8$ at centromeres after anaphase I.

Kitajima et al. show that Bub1 is, in fact, required to recruit Sgol to centromeres, suggesting a connection between the protection of Rec 8 and the spindle checkpoint. One possibility is that the lack of tension between sister centromeres in meiosis I - resulting from their enforced attachment to the same spindle pole - activates Bub1, which then recruits Sgo1. Such a signal would have to differ from that normally associated with a lack of inter-centromere tension; otherwise, division would stop 9

Broadening their findings, Kitajima et al. found that fission yeast contains a protein related to Sgo1, which they call Sgo2. This protein is located at centromeres in mitotic cells, and again the association depends on Bub1. Its function is unclear, although deleting it results in viable cells with a higher rate of chromosome loss. In addition, there is a single Sgo protein, Sgo1, in budding yeast, which seems to behave similarly to fissionyeast Sgo1 and Sgo2. The authors also identified proteins with regions of reasonable similarity in multicellular organisms. One of these proteins is the fruitfly Mei-S332 — so it seems that this protein's closely guarded secret is that it protects meiotic cohesion after all.

Our understanding of the role of Sgol in meiotic chromosome segregation has been confirmed and extended by three additional studies in both fission ${ }^{11}$ and budding ${ }^{12,13}$ yeast. Further insight has been provided into the role of fission yeast Sgo2 that localizes with meiotic centromeres ${ }^{11}$. It seems to be required in meiosis I to orient the unified sister-centromeres so that they only engage microtubules protruding from the same spindle pole. Thus, cells with Sgo2 deleted exhibit a primary defect in the segregation of chromosomes during meiosis I (ref. 11). The combined meiotic phenotype of cells lacking both Sgol and Sgo2 is essentially identical to that described for cells with no Bub1 (ref. 10). This is consistent with Bub1 being required to recruit these important determiners of chromosome behaviour to meiotic centromeres.

Returning to the prevalence of chromosome-segregation defects in ageing human eggs: could one contributing factor be a premature loss of the protective influence of Sgol during meiosis I, resulting in aberrant chromosome segregation in meiosis II? Sgo1 itself is usually degraded during anaphase I (ref. 3), but perhaps its levels also decline stochastically with maternal age in humans. This would, however, account only for the proportion of aberrations that are caused by defective meiosis II - and these are few compared with those occurring in meiosis I (refs 1, 2). Nonetheless, the discovery of the Sgo proteins should prompt further investigations that might provide insight into defects caused by aberrant numbers of human chromosomes.

Robin Allshire is at The Wellcome Trust Centre for Cell Biology, University of Edinburgh,

Edinburgh EH9 3JR, UK.

e-mail: robin.allshire@ed.ac.uk

1. Hassold, T. \& Hunt, P. Nature Rev. Genet. 2, 280-291 (2001).

2. Champion, M. \& Hawley, R. S. Nature Cell Biol. 4 (Suppl.), s50-s60 (2002).

3. Kitajima, T. S., Kawashima, S. A. \& Watanabe, Y. Nature 427, 510-517 (2004).

4. Rieder, C. L. \& Cole, R. J. Cell. Sci. 112, 2607-2613 (1999).

5. Petroncki, M., Siomos, M. F. \& Nasmyth, K. Cell 112, 423-440 (2003).

6. Watanabe, Y. \& Nurse, P. Nature 400, 461-464 (1999).

7. Klein, F. et al. Cell 98, 91-103 (1999).

8. Kerrebrock, A. W., Moore, D. P., Wu, J. S. \& Orr-Weaver, T. L. Cell 83, 247-256 (2004).

9. Musacchio, A. \& Hardwick, K. Nature Rev. Mol. Cell Biol. 3, 731-741 (2002)

10. Bernard, P., Maure, J. F. \& Javerzat, J.-P. Nature Cell Biol. 3, 522-526 (2001).

11. Rabitsch, K. P. et al. Curr. Biol. (in the press).

12. Katis, V., Galova, M., Rabitsch, K. P., Gregan, J. \& Nasmyth, K. Curr. Biol. (in the press).

13. Marston, A. L., Tham, W.-H., Shah, H. \& Amon, A. Science published online doi:10.1126/science.1094220 (2004). 